\title{
$\bullet$ Histomorphological Spectrum of Neoplastic Lesions of Kidney with a Brief Review of Literature
}

\section{IJCRR}

Section: Healthcare

ISI Impact Factor

(2019-20): 1.628

IC Value (2019): 90.81 $\operatorname{SJIF}(2020)=7.893$

(c) (i) (8)

Copyright@IJCRR

\section{Nalini Modepalli ${ }^{1}$, Jyothi Anantharaj ${ }^{2}$, Naveen Shivappa ${ }^{3}$, Shwetha Basavaraj", Sakshi Barve ${ }^{4}$}

'Associate Professor, Department of Pathology, Rajarajeswari Medical College and Hospital, Kambipura, Mysore Road, Bangalore, India; ${ }_{2}^{2}$ Professor, Department of Pathology, Rajarajeswari Medical College and Hospital, Kambipura, Mysore Road, Bangalore, India; ${ }^{3}$ Professor, Department of General Surgery, Rajarajeswari Medical College and Hospital, Kambipura, Mysore Road, Bangalore, India; ${ }^{4}$ Tutor, Department of Pathology, Rajarajeswari Medical College and Hospital, Kambipura, Mysore Road, Bangalore, India.

\section{ABSTRACT}

Introduction: Renal tumours are an important cause of morbidity worldwide. They include a diverse spectrum and the recent advances in the field of renal neoplasia has given a better understanding of the morphological and molecular characteristics leading to the new Vancouver classification and the new WHO classification of Renal tumours.

Objective: To study the histomorphological features of various renal tumours. To classify them as per the new WHO classification of renal Tumors and its correlation with the clinical findings.

Methods: A cross-sectional study of renal tumours diagnosed on the nephrectomy specimens received between January 2017 to December 2019 was carried out to study the various morphological types.

Results: Of the 39 nephrectomy specimens received during the study period, 15 were neoplastic and 24cases were non-neoplastic. Among neoplastic, 4 (26.6\%) cases were benign and 11cases(73.3\%) were malignant tumours, of which clear cell Renal Cell Carcinoma was the most common. Furhmann nuclear grade II was more frequently encountered in our study. The most common site was the upper pole of the kidney. The most common age of presentation was in the 7 th decade.

Conclusion: Reclassification of renal tumours based on their molecular, clinical and pathological features and the emergence of new entities has lead to a better understanding of renal neoplasms and also aids accurate diagnosis of these tumours leading to better management strategies.

Key Words: Kidney, Neoplasms, Morphology, Benign, Malignant

\section{INTRODUCTION}

The kidneys are paired, bean-shaped organs located on either side of the vertebral column in the retroperitoneal space. They are dynamic organs serving as the main osmoregulatory system (fluid-electrolyte balance) in humans. In addition to maintaining fluid homeostasis, they also act as an endocrine organ (secrete hormones - prostaglandins, and regulating vitamin D metabolism) control Red blood cell production by secreting hormone erythropoietin and regulate blood pressure through enzyme renin. ${ }^{1}$ The renal parenchyma though subjected to repeated trauma/insults of the noxious environment are the last to respond. They can be involved in various pathological processes, which can be diagnosed on biopsies and nephrectomy specimens. Various disease processes affect the kidneys, some result- ing in permanent damage leading to surgical removal of the organ.

Nephrectomy is a common procedure in surgical practice and is of many types; partial, total and radical nephrectomy. A standard radical nephrectomy specimen consists of the entire kidney including the calyces, pelvis, variable length of the ureter, entire perirenal fatty tissue to the level of Gerota's fascia and variable lengths of the major renal vessels at the hilus. The adrenal gland is usually removed en bloc with the kidney. Regional lymphadenectomy is not generally performed even with a radical nephrectomy. ${ }^{2} \mathrm{~A}$ partial nephrectomy specimen may vary from a simple enucleation of the tumour to part of a kidney containing variable portions of the calyceal or renal pelvic collecting system. The perirenal fat immediately overlying the resected

\section{Corresponding Author:}

Dr. Nalini Modepalli, Associate Professor, Department of Pathology, Rajarajeswari Medical College and Hospital, Kambipura, Mysore Road, Bangalore, India; Contact Number: +919902825235; Email: doctor.nalini@gmail.com

ISSN: $2231-2196$ (Print)

Received: 10.09 .2020
ISSN: 0975-5241 (Online)

Revised: 15.11 .2020
Accepted: 20.12 .2020
Published: 12.04 .2021 
portion of the kidney but not to a level of Gerota's fascia is usually included. ${ }^{2}$

Radical nephrectomy indicated in end-stage renal disease, treatment of renovascular hypertension from non-correctable renal artery disease, or in severe unilateral parenchymal damage resulting from nephrosclerosis, pyelonephritis, Xanthogranulomatous pyelonephritis, vesicoureteric reflux, and congenital dysplasia. It is the treatment of choice in renal cell carcinomas. ${ }^{3}$ Percutaneous image-guided biopsy of renal masses is a safe and accurate procedure, being increasingly used to differentiate between benign and malignant entities to avoid unnecessary surgery. ${ }^{4}$ In the last few years, there has been a growing interest in nephron-sparing surgery or partial nephrectomy to treat the selected cases of localized renal cell carcinoma (RCC) by open or laparoscopic approach. Renal cancer is the 9th most common cancer in men accounting for $2.5 \%$ of total malignancies. WHO (2018). ${ }^{5}$

The histological classification of RCCs is extremely important, considering the significant implications of the subtypes in the prognosis and treatment of these tumours. ${ }^{6}$ In 2004 World Health Organization (WHO) published a histological classification of renal tumours. ${ }^{7}$ In 2012, the International Society of Urological Pathology (ISUP) organized a conference in Vancouver, Canada which gave a new classification, referred to as "2012 ISUP Vancouver classification" (2012 ISUP). On the foundation of 2012 ISUP classification, 2016 World Health Organization (WHO) renal tumour classification was published after considering new knowledge about pathology, epidemiology, and genetics. ${ }^{3}$ Molecular and morphologic interrogation has driven a much-needed reexamination of renal cell carcinoma (RCC). The latest 2016 World Health Organization classification now recognizes 12 distinct RCC subtypes, as well as several another emerging/ provisional RCC entities. (expanded from 4 subtypes in the 1997 Heidelberg classification to 12 recognized subtypes in 2016) ${ }^{8}$

Important changes from previously classified renal tumour types, to the new 2016 WHO classification refers to subtypes that have been named based on predominant cytoplasmic features (eg, clear cell and chromophobe renal cell carcinomas [RCCs]), architectural features (eg, papillary RCC), anatomic location of tumours (eg, collecting duct and renal medullary carcinomas), and correlation with a specific renal disease background (eg, acquired cystic disease-associated RCCs) as well as molecular alterations pathognomonic for RCC subtypes (eg, MiT family translocation carcinomas and succinate dehydrogenase [SDH]-deficient renal carcinomas) or familial predisposition syndromes (eg, hereditary leiomyomatosis and RCC [HLRCC] syndrome- associated RCC). ${ }^{9}$

Emerging/provisional entities are TCEB1-mutated RCC/ RCC with angioleiomyoma-like stroma/RCC with leiomyomatosis stroma, RCC associated with ALK gene rearrange- ment, Thyroidlike follicular RCC and RCC in survivors of NB. ${ }^{8}$ The Fuhrman system is the most frequently used grading system but has its limitations. The four-tiered WHO/ ISUP grading system is recommended by the WHO . ${ }^{9} \mathrm{How}-$ ever, this too has been validated as a prognostic indicator only for clear cell and papillary RCC and not for other morphotypes of renal cell neoplasms.

2016 WHO classification describes several new and emerging entities along with the previously recognized and also the provisional RCC types. This mandates the surgical pathologists to integrate clinical, radiologic, gross, and microscopic findings to successfully navigate challenging differential diagnoses in RCC classification. Unusual morphologic features ( clear cytoplasm, papillary architecture, and eosinophilic (oncocytic) cytoplasm) seen in various subtypes need to be kept in mind in our approach to various RCC's. Hence we intended to study the morphological subtypes of the renal tumour using the new WHO classification.

\section{MATERIALS AND METHODS}

We conducted a cross-sectional/observational study on all the nephrectomy specimens received in the Central laboratory, Department of Pathology (Histopathology division) Rajarajeswari Medical College and Hospital, Bangalore, India from Jan 2017 to Dec 2019.

Inclusion criteria: Nephrectomy Specimens received in a central lab, department of Pathology, Rajarajeswari Medical College and Hospital which on histopathological examination was diagnosed as Renal tumour listed under the WHO classification of tumours of kidney were included in this study.

Exclusion criteria: The non-neoplastic lesions and metastatic tumours of the kidney were excluded from the study.

Study Design: Specimens were received in 10\% formalin. Essential clinical details including patient's identification, age, sex, clinical data, investigations such as CT scan, USG, other relevant investigations were noted from the test request form. After proper fixation, weight and dimensions were recorded and the gross findings including examination of the capsule, external surface and features on the cut section were noted. Sections for histopathological examination from a lesion suspicious of tumour were taken from Tumor proper (multiple bits), Tumor with renal capsule, Tumor with Gerota's fascia, hilar structures, Renal sinus, Renal pelvis, vessels, ureter and surgical margins. Lymph nodes(if any) and sections from the adjacent kidney are taken to look for associated features in the adjacent renal parenchyma. Tissue was processed, embedded, sections were cut and stained by Haematoxylin and Eosin stain and submitted for histopathological examination. Microscopic features were noted and final reports were signed out as per the CAP protocol. 


\section{RESULTS}

A total of 39 nephrectomy specimens were received during the study period, out of which $15 \operatorname{cases}(38.4 \%)$ were neoplastic and 24cases (61.6\%)were non-neoplastic. Among neoplastic, $4(26.6 \%)$ cases were benign and 11 cases(73.3\%) were malignant tumours. Flank pain (12 cases) was the most common clinical presentation in Renal tumours followed by abdominal mass(11 cases). The highest percentage of patients belong to the 61-70years age group $(46.6 \%)$ with female preponderance. Tumours were more common on the left than the right side with a predilection to the upper pole (40\%). Clear cell renal cell carcinoma was a more common malignant tumour and angiomyolipoma was the common one among the benign ones. Of the 10 Renal cell carcinomas, Fuhrman nuclear grading 2 was marginally more common(4 cases).

\section{DISCUSSION}

The kidney is by far a very resilient organ capable of withstanding the repeated stimuli by various disease processes. Once permanent irreversible damage sets in, a nephrectomy is indicated. In our 2 year study, 15 primary renal neoplastic lesions were identified among the 39 nephrectomies received in that period. Malignant tumours accounted for $73.3 \%$ of cases which were concurrent with the previous studies. ${ }^{10-13}$ The mean age in the present study is $42.7 \mathrm{yrs}$ with the majority of patients in the age group of $60-70 \mathrm{yrs}$, the youngest being 1year old with Wilms tumour. These findings were consistent with a study conducted previously. ${ }^{14}$

There was female preponderance in our study with a male: female ratio of 1:1.2. This finding was in correlation with studies $^{3,15}$ but most of the other studies showed a male preponderance. The left kidney showed a higher incidence of neoplastic lesions in our study as was in the studies. ${ }^{16,17}$ Most of the patients presented with flank pain (12 cases) and abdominal mass (11 cases), a finding similar to the study conducted by Bharti et al. ${ }^{18}$ Grossly, most of the tumours were situated in the upper pole (40\%) in our study compared to Popat et al. ${ }^{19}$

The most common malignant tumour was RCC (46.6\%), among which clear cell RCC was the most common histologic variant, consistent with the available literature. This was followed by chromophobe type RCC (2/11 malignant tumours) followed by one case each of Papillary RCC, Clear cell papillary RCC (with low-grade clear cells in tubules and papillae), Multicystic RCC. Clear cell papillary renal cell carcinoma (CCP-RCC) is a recently proposed, distinctive, uncommon and indolent renal epithelial neoplasm, thought to arise in end-stage renal disease..$^{20}$ They are often small, well-circumscribed, and encapsulated, accounting for $1-4 \%$ of all resected renal tumours. These tumours have immunophenotype and molecular profiles distinct from those of clear cell and papillary RCCs. ${ }^{21}$

CCP-RCC is composed of bland clear epithelial cells arranged in tubules and papillae, with linear nuclear alignment away from the basement membrane and low Fuhrman nuclear grade. Most tumours are WHO I International Society of Urological Pathology (ISUP) grade 1 or $2 .{ }^{21}$ Clear cell RCC rarely has papillary architecture while papillary RCC only rarely contains clear cells. Primary RCCs with both papillary architecture and cells with clear cytoplasm are diagnostically challenging for pathologists. Clues to the diagnosis of CCPRCC include unique subnuclear cytoplasmic clearing, low nuclear grade, prominent smooth muscle stroma, and in some cases an end-stage background kidney. The morphology and typical IHC pattern of positive CK7 and CAIX but negative CD10 and AMACR should lead to accurate classification. The absence of $3 p$ loss or trisomy of chromosomes 7 and 17 support the above diagnosis. Despite these tools, some RCCs with papillary architecture and clear cells do not fit into any of the described entities and currently remain unclassified.22

One case of Clear cell RCC showed sarcomatoid features with $50-60 \%$ of cells arranged in solid sheets of spindle cells with high pleomorphism and high nuclear grade (Fuhrman grade 4). Sarcomatoid RCC (sRCC) is no longer considered a separate tumour type as it can occur with all histologic subtypes. ${ }^{23}$ It is now recognized as a high-grade undifferentiated/ dedifferentiated component of RCC, characterized by both epithelial and mesenchymal differentiation. These components share a common cell of origin as suggested by their genomic features. ${ }^{24}$ The sarcomatoid pattern may result from the activation of a distinct sarcomatoid stem cell within the tumour. The average incidence is $8 \%$ among all RCCs. ${ }^{24}$ These tumours contain features similar to sarcomas, with spindle-like cells, high cellularity, and cellular atypia. These rare tumours show variable amounts of carcinoma elements and barely any classic RCC areas. Hence IHC using epithelial and mesenchymal is mandatory to distinguish sarcomas from SRCC - sarcomatoid areas express cytokeratin AE1/AE3 and are negative for mesenchymal markers, such as desmin and actin. ${ }^{23}$

These tumours are aggressive, progress rapidly and carry a poor prognosis, irrespective of the underlying RCC subtype. These patients have a reported median survival time of 4-9 months after diagnosis. Major prognostic factors include the proportion of sarcomatoid components, pathological stage, tumour necrosis, tumour size and genetic factors. Distant metastases are common in the lungs and bones. ${ }^{24}$ One case of Wilms tumour in a one-year-old was reported in the study. In the present study Furhman grade -II was seen more commonly among the Renal cell carcinomas as seen earlier. ${ }^{3}$ Among the 4 benign tumours encountered in our study, 3 
were Angiomyolipomas and one oncocytoma, these findings were similar to the results reported. ${ }^{3,13}$ Nine cases showed associated findings in the adjacent renal parenchyma which included cortical cysts, chronic glomerulonephritis and chronic pyelonephritis predominantly indicating an ongoing chronic process.

\section{CONCLUSION}

Histological classification of renal neoplasia has undergone significant changes with various new entities with distinct clinical, pathological and molecular characteristics. Histologic subtype of RCC strongly correlates with prognosis, clinical implications and therapeutic strategies, underscoring the importance of accurate diagnosis. Attention to morphology and targeted immunohistochemical panel allow accurate classification of most RCCs. In others, cytogenetic and molecular findings, although expensive can establish the diagnosis. Our study provides an insight into various commonly encountered histomorphological patterns of kidney tumours and their associated features.

\section{ACKNOWLEDGEMENT}

Authors acknowledge the immense help received from the scholars whose articles are cited and included in references to this manuscript. The authors are also grateful to authors/ editors/publishers of all those articles, journals and books from where the literature for this article has been reviewed and discussed.

Conflict of Interest: The authors have no conflicts of interest to declare.

\section{Source of Funding: NIL}

Authors and their contribution: Nalini M, Jyothi A Raj, and Naveen $\mathrm{S}$ have contributed to the conception and design of the study and have authored the main manuscript. Shwetha B and Sakshi Barve have collected clinical and pathological data. All authors have contributed to the literature review, editing, and approval of the final draft of the manuscript. All authors agree to be accountable for the content of this manuscript and its submission to the International Journal of Current Research and review.

\section{REFERENCES}

1. Alpers CE, Chang A. Robbins and Cotran Pathologic basis of disease. 9e South Asian edition. India: Reed Elsevier: 2014. Chapter 20, the Kidney: p 897-898.

2. Srigley JR, Zhou M, Allan R, Amin MB, Campbell SC, Chang A, et al. Protocol for the Examination of Specimens from $\mathrm{Pa}-$ tients with Invasive Carcinoma of Renal Tubular Origin. Col- lege of American Pathologists ; June 2017. 15p. available from www.cap.org/cancerprotocols version :kidney 4.0.1.0

3. Aiman A, Singh K, Yasir M. Histopathological spectrum of lesions in nephrectomy specimens: A five-year experience in a tertiary care hospital. J Sci Soc 2013;40:148-54.

4. Sahni VA, Silverman SG. Biopsy of renal masses: when and why. Cancer Imaging. 2009;9(1):44.

5. Bray F, Ferlay J, Soerjomataram I, Siegal RL, Torre LA, Jemal A. Global Cancer Statistics 2018: GLOBOCAN Estimates of Incidence and Mortality Worldwide for 36 Cancers in 185 Countries. Cancer J Clin 2018;68:394-424.

6. Muglia VF, Prando A. Renal cell carcinoma: histological classification and correlation with imaging findings. Radiol Bras 2015;48(3):166-174.

7. Hes O, Compérat EM, Rioux-Leclercq N, Kuroda N. The 2012 ISUP Vancouver and 2016 WHO classification of adult renal tumors: changes for common renal tumors. Diagn Histopathol 2016;22(2):41-46.

8. Udager AM, Mehra R. Morphologic, Molecular, and Taxonomic Evolution of Renal Cell Carcinoma - A Conceptual Perspective With Emphasis on Updates to the 2016 World Health Organization Classification. Arch Pathol Lab Med 2016;140:1026-1037.

9. Moch H, Cubilla AL, Humphrey PA, Reuter VE, Ulbright UM. 2016 WHO Classification of Tumours of the Urinary System and Male Genital Organs-Part A: Renal, Penile, and Testicular Tumours. Eur Urol 2016;70:93-105.

10. Vinay KS, Sujatha S. Histopathological Spectrum of Nephrectomy Specimens: Single Center Experience. Biomed J Sci Tech Res 2018;6(3).

11. Reddy KD, Gollapalli SL, Chougani S, Sidagam S, Mohmmed AK, Bommanna A. A clinic-morphological spectrum of nephrectomy specimens-an experience from a tertiary care hospital. Int J Health Sci Res 2016;6(11):67-72.

12. Bashir N, Bashir Y, Shah P, Bhat N, Salim O, Samoon N et al. Histopathological study of renal tumors in resected Nephrectomy specimens-An experience from teritary care centre. Nat J Med Res 2015;5(1):25-29.

13. Ghafoor AS. Kareem A, Bashar A. Hassawi, Ziyad Ahmed. Nephrectomy. A clinicopathological study. J Am Sci 2015;11(8):97101.

14. Narang VI, Garg BH, Walia AS, Sood NE, Malhotra VI. Histomorphological Spectrum of Nephrectomy Specimens-A Tertairy Care Centre Experience. Natl J Lab Med 2016 Apr;5(2):51-4.

15. Ibrahim SS, Dhanabalan RT, Sankari S, Shanmuganathan LK, Thandavarayan P, Ramalingam S, et al. A histopathological review of nephrectomy specimens received in a referral center in South Tamil Nadu. Age 2016;1(10).

16. Chaitra B, Prema LP, Tejeswini V, Haritha O, Anusha M. Histopathology of nephrectomy specimens: A ten year south Indian tertiary hospital-based study. J Diagn Pathol Oncol 2018;4(3):232-236.

17. Ajmera S, Ajmera R. Histopathological spectrum of lesions in nephrectomies- A five year study. Int J Sci Res 2017;6(7):44-46.

18. Thaker BD, Singh K. A histopathological review of Nephrectomy specimens Received in a Tertiary care hospital-A retrospective study. J Med Sci Clin Res 2017;5(6):23807-23810.

19. Popat VC, Kumar MP, Udani D, Mundra MP, Vora DN, Porecha MM. A study on culprit factors ultimately demanding nephrectomy. Internet J Urol 2010;7(1):1-8.

20. Kuroda N, Ohe C, Kawakami F, Mikami S, Furuya M, Matsuura $\mathrm{K}$ et al. Clear cell papillary renal cell carcinoma: A review. Int J Clin Exp Pathol 2014;7(11):7312-7318.

21. Srigley JR, Cheng L, Grignon DJ, Tickoo SK. Clear cell papillary renal cell carcinoma in chapter 1: Tumors of the kidney. Moch H, Humphrey PA, Ulbright TM, Reuter VE (Eds): WHO 
Classification of Tumours of the Urinary System and Male Genital Organs (4th edition) IARC: Lyon 2016. pp 40-41.

22. Ross H, Martignoni G, Argani P. Renal Cell Carcinoma with Clear Cell and Papillary Features. Arch Pathol Lab Med 2012;136:391-399.

23. Shuch B, Bratslavsky G, Linehan WM, Srinivasan R. Sarcomatoid Renal Cell Carcinoma: A Comprehensive Review of the Biology and Current Treatment Strategies. Oncologist 2012;17:46-
54.

24. Liang X, Liu Y, Ran P, Tang M, Xu C, Zhu Y. Sarcomatoid renal cell carcinoma: a case report and literature review. BMC Nephrology 2018;19:84.

Table 1 : Distribution of neoplastic and non-neoplastic lesions.
Total no.of nephrectomies Received

Non neoplastic

Neoplastic
Benign

Malignant

\section{9}

$24(61.5 \%)$

$4(10.2 \%)$

$11(28.2 \%)$

Table 2 : Clinical presentation

\begin{tabular}{lc} 
Symptoms & No. of Cases \\
Flank pain & 12 \\
Abdominal mass & 11 \\
Weight loss & 05 \\
Haematuria & 05 \\
Fever & 02 \\
Oliguria & 02 \\
\hline
\end{tabular}

Table 3: Distribution of neoplastic nephrectomy specimens according to age

\begin{tabular}{lc} 
Age Range & No. of Cases \\
$0-10$ & $1(6.6)$ \\
$11-20$ & 0 \\
$21-30$ & 0 \\
$31-40$ & $3(20)$ \\
$41-50$ & $1(6.6)$ \\
$51-60$ & $3(20)$ \\
$61-70$ & $7(46.8)$ \\
Total & 15 \\
\hline
\end{tabular}

Table 4 : Distribution of neoplastic lesions according to side

$\begin{array}{lcc} & \text { LATERALITY } & \\ & \text { RIGHT } & 2 \\ \text { BENIGN } & 2 & 6 \\ \text { MALIGNANT } & 5 & (56.4 \%) \\ \end{array}$


Table 5 : Histopathological spectrum of neoplasms.

\begin{tabular}{lcc} 
& NO. OF CASES & PERCENTAGE \\
MALIGNANT TUMORS & 11 & $73 \cdot 3$ \\
Clear cell Renal cell carcinoma & 5 & $33 \cdot 3$ \\
Chromophobe renal cell carcinoma & 2 & $13 \cdot 4$ \\
Papillary Renal cell carcinoma & 1 & 6.6 \\
Clear cell papillary Renal cell carcinoma & 1 & 6.6 \\
Multicystic Renal cell carcinoma & 1 & 6.6 \\
Wilms Tumor & 1 & 6.6 \\
BENIGN TUMORS & 4 & 26.7 \\
Oncocytoma & 1 & 6.6 \\
Angiomyolipoma & 3 & 20.1 \\
\hline
\end{tabular}

Table 6 : Furhman nuclear grading of Renal cell carcinoma

\begin{tabular}{lc} 
Nuclear grading & No.of cases \\
I & $2(20 \%)$ \\
II & $4(40 \%)$ \\
III & $3(30 \%)$ \\
IV & $1(10 \%)$ \\
\hline
\end{tabular}

Table 7 : Distribution of tumors according to location

\begin{tabular}{lc} 
Location & Number of cases \\
Entire kidney & $2(13 \cdot 3)$ \\
Upper pole & $6(40)$ \\
Mid region & $4(26.6)$ \\
Lower pole & $3(20)$ \\
\hline
\end{tabular}

Table 8: Associated findings in neoplastic lesions

\section{Associated findings}

Cortical cyst

Chronic glomerulonephritis

Chronic-pyelonephritis

Glomerulo-sclerosis

Interstitial inflammation

\section{In 9 cases}

$3(33 \cdot 3 \%)$

$2(22.3 \%)$

$2(22.3 \%)$

$1(11.1 \%)$

$1(11.1 \%)$ 

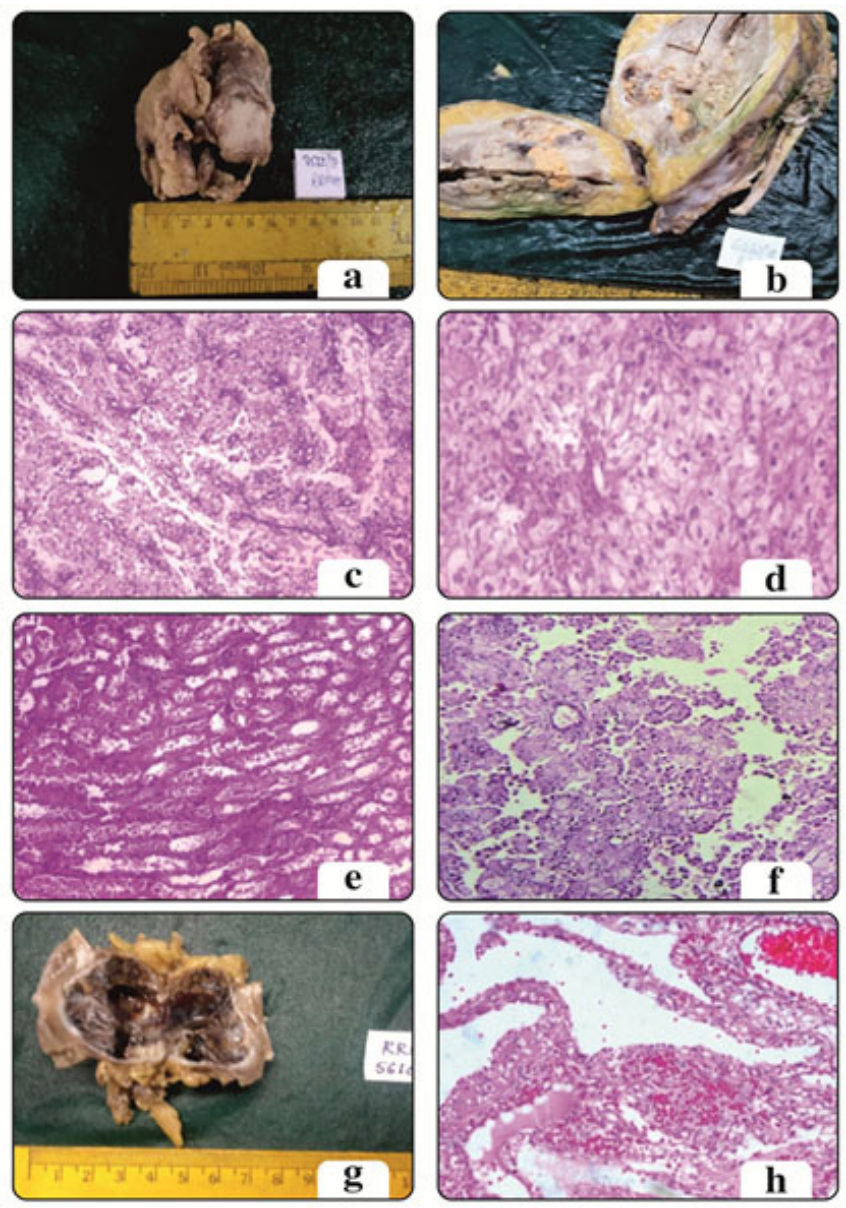

Figure 1: Renal Cell carcinoma. (a) Cut section shows a grey white mass in the midpole. (b) Renal Cell Carcinoma - clear cell type (with sarcomatoid features). Cut section shows variegated appearance with grey white solid and necrotic areas. (c) Renal cell carcinoma, Clear cell type Photomicrograph shows solid ,alveolar pattern of cells with clear eosinophillic cytoplasm with Fuhrman's Nuclear grade 2, H\&E (x20). (d) Renal cell carcinoma clear cell type. H\&E x40 magnification (e) Renal cell carcinoma, Papillary type. H\&E x40 magnification. (f) Renal cell carcinoma,chromophobe type H\&E x40 magnification. (g) Multicystic RCC. Cut section: multiloculated cystic mass and thin septae in between the cysts. (h) Multicystic RCC Photomicrograph shows cysts of varying sizes separated by septae containing tumor cells, $H \& E(X 10)$.
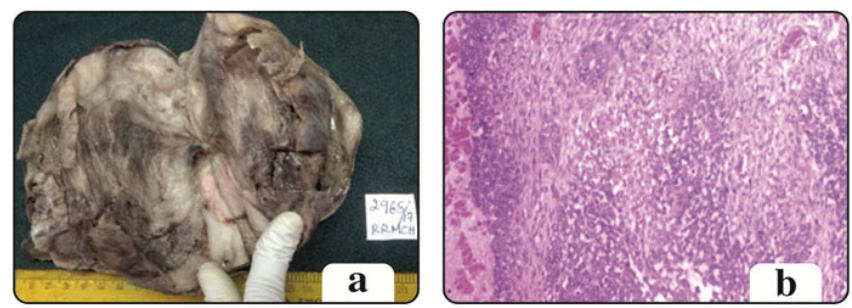

Figure 2: Wilm's tumor. (a) External surface shows large kidney with adherent capsule. Cut section: Large mass with solid greyish white areas and cystic areas. (b) Photomicrograph of Blastemal, stromal and epithelial component in Wilm's tumor $(H \& E, x 40)$.
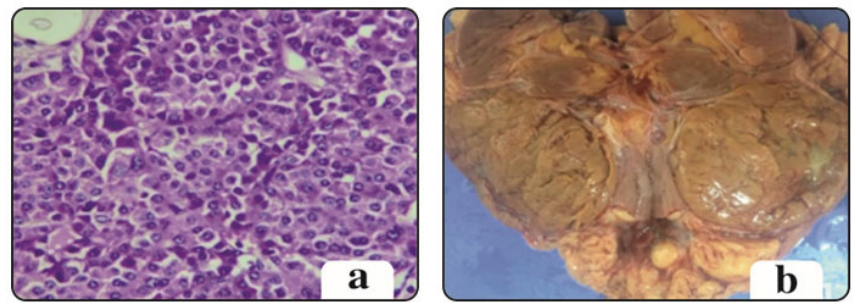

Figure 3: Oncocytoma. (a) Microscopy shows nesting and alveolar pattern of round to polygonal cells with abundant eosinophillic granular cytoplasm and central nuclei.(H and E 40x). (b) Cut surface shows well circumscribed, solid ,homogenous, mahagony mass with central scar.
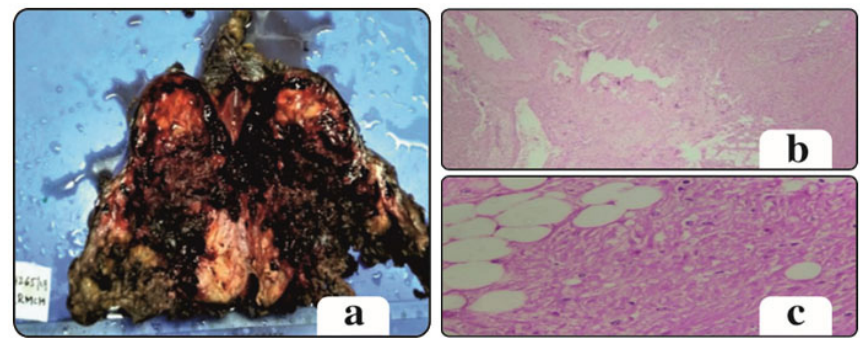

Figure 4: Angiomyolipoma. Cut surface shows haemorrhagic, gray white and gray yellow areas. (b and c) Microscopy shows mature adipocytes, blood vesssels and smooth muscle components. (H and E 10X (c) H\&E 40X. 